\title{
Efecto del Uso de la Estrategia de Enseñanza Aprendizaje Basado en Problemas (ABP) en el Desarrollo de las Destrezas de Comprensión y Análisis de la Estadística Descriptiva
}

\author{
Effect of the Use of the Problem Based Learning Teaching \\ Strategy in the Development of Comprehension Skills and \\ Analysis of Descriptive Statistics
}

\author{
Luz Celenia Colón Ortiz * \\ Jaime Ortiz-Vega \\ Pontificia Universidad Católica de Puerto Rico, Puerto Rico
}

\begin{abstract}
La escuela debe capacitar a los estudiantes para que sean útiles a la sociedad en la cual conviven. Los informes del perfil de los estudiantes del Departamento de Educación de Puerto Rico (DEPR) para los años 2015-2018, demuestran que estos no dominan las destrezas básicas en la materia de matemáticas. Los resultados de las pruebas estandarizadas demuestran que el dominio de tales destrezas ha ido disminuyendo. En este artículo se detalla información respecto a los hallazgos de una investigación cuyo propósito fue determinar el efecto del uso de la estrategia de enseñanza Aprendizaje Basado en Problemas (ABP) en el desarrollo de las destrezas de comprensión y análisis de la estadística descriptiva en estudiantes de décimo grado en una escuela pública de un distrito escolar de la región central de Puerto Rico. Se utilizó un enfoque cuantitativo con diseño cuasi experimental. La población se seleccionó por disponibilidad. La muestra quedó constituida por 48 estudiantes de los cuales 25 formaron el grupo control (GC) y 23 el grupo experimental (GE). La asignación del GC y del GE se realizó aleatoriamente. Los datos se recopilaron a través de una prueba sobre estadística descriptiva. La información recopilada se analizó mediante una prueba $t$ para grupos independientes, prueba $t$ pareada y regresión lineal. Los resultados demostraron que el uso del ABP tuvo un efecto significativo en el desarrollo de las destrezas de comprensión y análisis de la estadística descriptiva. Los estudiantes del GE obtuvieron puntuaciones más altas en la posprueba que los estudiantes del GC.
\end{abstract}

Palabras clave: Aprendizaje basado en problema; Estrategia de enseñanza; Destrezas de comprensión y análisis; Estadística descriptiva; Pruebas estandarizadas.

\begin{abstract}
School must prepare students in an integral way in order to serve and contribute to the improvement of society. The profile report of the Department of Education of Puerto Rico (DEPR) during 2015-2018 revealed that students do not mastered math basic skills. A decrease in standardized tests results is evidenced. This article presents the results of an investigation to determine the effect of the Problem Base Learning (PBL) teaching strategy in the development of comprehension and analysis skills in descriptive statistics in tenth grade students at a public school in the central region of Puerto Rico. A quantitative, quasi-experimental approach was used. The sample was selected by availability, for a total of 48. 25 students were in the control group (CG) while 23 were in the experimental group (EG). Data was obtained through a pretest and posttest about descriptive statistic. The data was analyzed using descriptive statistic (independent test $t$, paired test $t$ and lineal regression). Results showed that the use of the PBL strategy had a positive effect in the development of the comprehension and analysis skills in descriptive statistic. Students from the experimental group performed higher in the posttest than those from the control group.
\end{abstract}

Keywords: Problem based learning; Teaching strategy; Comprehension and analysis skills; Descriptive statistic; Standardized tests.

*Contacto: 1colonortiz1@pucpr.edu

issn: 1989-0397

www.rinace.net/riee/

https://revistas.uam.es/riee
Recibido: $\quad 01$ de febrero de 2019

$1^{\text {a }}$ Evaluación: 03 de marzo de 2019

$2^{\text {a }}$ Evaluación: 29 de abril de 2019

Aceptado: $\quad 27$ de mayo de 2019 


\section{Introducción}

El currículo de matemática en las escuelas del Departamento de Educación de Puerto Rico (DEPR) (DEPR, 2016) ha sido transformado con el pasar de los años. Según el Marco Curricular del Programa de Matemáticas del DEPR, (DEPR, 2016) es fundamental desarrollar las destrezas y competencias que permitan a los estudiantes aprender mientras hacen, y en el proceso, aprender unos de otros. En la función de responder a las necesidades y exigencias de la sociedad contemporánea, el referido documento establece un currículo cuyo fin es formar seres humanos capaces de enfrentarse a los cambios y para que puedan lidiar con las situaciones del diario vivir y tomar decisiones eficaces en un mundo competitivo en el cual les toca vivir.

Hasta mayo de 2015 el DEPR utilizó las Pruebas Puertorriqueñas de Aprovechamiento Académico (PPAA) para medir el desarrollo de las destrezas matemáticas de los estudiantes. De acuerdo con los resultados de estas pruebas, el 70,36\% de los estudiantes demostraron no tener, al menos, dominio satisfactorio en las destrezas medidas. Dado esto, el DEPR instituyó un Plan de Transformación Académica, llamado META PR, con visión longitudinal y un nuevo enfoque de evaluación de estudiantes que sustituyó las PPAA.

En el cuadro1 se presenta información relacionada con el desempeño de los estudiantes del DEPR en las PPAA (2013 - 2015) y META PR (2016-2017) en la materia de matemáticas durante los años académicos 2013, 2014, 2015, 2016, y 2017.

Cuadro 1. Porcentajes obtenidos en la Pruebas PPAA y META PR, en la materia de matemáticas (DEPR, 2018)

\begin{tabular}{cccccc}
\hline ESTUDIANTES & $\mathbf{2 0 1 3}$ & $\mathbf{2 0 1 4}$ & $\mathbf{2 0 1 5}$ & $\mathbf{2 0 1 6}$ & $\mathbf{2 0 1 7}$ \\
\hline 3ero & 66 & 70 & 76 & 74 & 76 \\
4to & 53 & 54 & 65 & 62 & 62 \\
5to & 40 & 44 & 47 & 45 & 48 \\
6to & 17 & 19 & 21 & 19 & 14 \\
7 mo & 9 & 8 & 8 & 10 & 11 \\
8vo & 10 & 10 & 7 & $34 \%$ & 10 \\
11mo & 10 & 10 & $34 \%$ & $33 \%$ \\
\hline Nivel Isla & & & & 39
\end{tabular}

Fuente: Elaboración propia.

Se desprende del cuadro 1, que, a partir de quinto grado, los porcientos de dominio de las destrezas medidas disminuyeron de forma substancial.

Como puede inferirse a través de la literatura presentada y de las pruebas a las cuales se hace referencia, la enseñanza de las matemáticas en el DEPR es un área académica de prioridad debido a la deficiencia que han mostrado los estudiantes en su desempeño en las PPAA y META PR. La misma muestra que existe la necesidad de crear y utilizar diferentes estrategias que ayuden a mejorar el desempeño de los estudiantes en la clase de matemáticas

Por lo anteriormente expuesto, se hace imperativo tomar acciones que permitan revertir esta situación. En la investigación cuyos resultados se resumen en esta lectura se pretendió determinar cuál es el efecto del uso de la estrategia de enseñanza Aprendizaje Basado en Problemas (ABP) en el desarrollo de las destrezas de comprensión y análisis en la estadística descriptiva. Esto, con el propósito de intentar mejorar la situación planteada respecto al desarrollo de las destrezas de razonamiento matemático. 
El Aprendizaje Basado en Problemas (ABP) se constituye como una estrategia viable para contrarrestar los problemas que conlleva la enseñanza tradicional. En tal sentido, Pantoja, y Covarrubias (2013) llevaron a cabo una investigación basada en dicha estrategia de enseñanza, que muestra la promoción de habilidades de pensamiento necesarias para el aprendizaje significativo. Se trabajó con estudiantes universitarios, en una situación que requirió análisis, solución y el uso de diferentes estrategias didácticas. Se utilizó un diseño cuasi experimental, con análisis estadísticos y cualitativos de los datos obtenidos. Los resultados reflejaron que el $\mathrm{ABP}$ es una estrategia de enseñanza que permite que el aprendizaje sea uno significativo en cuanto a su contenido.

Fuentes y Pérez (2013) llevaron a cabo una investigación cuyo objetivo fue determinar el efecto del ABP en el aprendizaje logrado por estudiantes en diferentes materias. La investigación fue una correlacionar comparativo con grupo histórico, donde participaron 29 estudiantes, de los cuales 14 fueron impactados con la estrategia ABP y 15 no fueron impactados con dicha estrategia, como grupo comparativo. La recolección de datos se realizó mediante encuesta, evaluación cualitativa del ABP, Cuestionario Honey-Alonso de Estilos de Aprendizaje y pruebas de conocimientos entre otros. Los resultados demostraron diferencia significativa en el rendimiento académico, en los estilos de aprendizajes y antecedentes académicos previos. Al tomar en cuenta el compromiso docente que perciben los estudiantes, los autores recomendaron el uso de la estrategia de enseñanza ABP con mayor frecuencia.

De otra parte, Carmona, Conesa y Ros (2014) llevaron a cabo una investigación cuyo objetivo fue determinar el efecto de la aplicación de la estrategia de enseñanza ABP en el proceso de enseñanza y aprendizaje de los estudiantes. Establecieron como hipótesis que existía efecto significativo en la aplicación del ABP como estrategia de enseñanza en el aprendizaje de los estudiantes. Para recopilar información, se llevó a cabo una encuesta a 83 estudiantes. Para analizar la información recopilada se utilizó estadística descriptiva. Los resultados indicaron que existe efecto positivo en la aplicación de la estrategia de enseñanza $\mathrm{ABP}$ en el proceso de enseñanza y aprendizaje de los estudiantes. Los investigadores concluyeron que participar en la estrategia de enseñanza ABP es una experiencia creativa y valorada por los estudiantes.

Por su parte, Soto, Figarella y González (2016) llevaron a cabo una investigación en la Universidad de Puerto Rico, Recinto de Río Piedras, para determinar cómo la estrategia de ABP promovía el desarrollo de la alfabetización crítica y de competencias en estudiantes de tercer grado, así como las destrezas de análisis, inferir, deliberar y cuestionar, entre otros. El marco teórico en el cual se sustentó la investigación fue el enfoque constructivista. En la investigación participaron 25 estudiantes, 15 niños y 10 niñas entre las de edades de siete a nueve años.

Para llevar a cabo la recopilación de datos se utilizaron observaciones participativas y no participativas, notas de campo, fotografías y materiales creados por los estudiantes durante el proceso para tomar acción sobre el problema que se les presentó. Según los autores, los resultados demostraron que tanto los niños como las niñas desarrollaron la destreza de cuestionamiento y análisis, habilidad en la toma de acción, capacidad para inquirir, para el diálogo y la deliberación. Además, concluyeron que la estrategia de ABP es una excelente herramienta para el desarrollo de las competencias ciudadanas y la alfabetización crítica para promover el desarrollo integral de los estudiantes. 
De manera similar, Delgado (2016) llevó a cabo una investigación para determinar el rol de la colaboración y el APB. La misma fue de tipo cualitativo y utilizó como diseño el estudio de caso con estudiantes de noveno grado de una escuela pública en Puerto Rico. Los participantes fueron 19 estudiantes, a quienes organizaron en grupos cooperativos de cinco, por tema de interés. Las técnicas que se utilizaron para la recopilación de los datos fueron: observaciones participativas, grupos focales y análisis de documentos. Para llevar a cabo el análisis de la información recopilada, se aplicó la Teoría de Actividad, que concentra la unidad de análisis en la actividad humana en un contexto particular. Tal análisis demostró que las interacciones entre pares y maestros, así como entre estudiantes y material didáctico son fundamentales en el proceso de enseñanza y aprendizaje. Una mayor interacción entre los estudiantes durante el proceso de planificar y desarrollar las tareas promueve una mejor comprensión de los conceptos según lo evidencia el uso de la estrategia ABP. Por lo tanto, la autora recomienda el uso de la estrategia ABP por parte de los maestros.

Para guiar la investigación se formuló el problema: ¿Cuál es el efecto del uso de la estrategia de enseñanza Aprendizaje Basado en Problemas (ABP) en el desarrollo de las destrezas de comprensión y análisis de la estadística descriptiva en los estudiantes de décimo grado en las escuelas públicas de un Distrito Escolar del centro de Puerto Rico?

\section{Fundamentación teórica}

La base teórica que explica el fenómeno bajo investigación es la teoría del aprendizaje significativo de Ausubel (2002). Además, se utilizó la Taxonomía de Bloom (1956) para desarrollar el proceso de enseñanza y aprendizaje en la sala de clases y evaluar el nivel de conocimiento adquirido por los estudiantes, ya que busca que lleguen a desarrollar habilidades que les permitan utilizar el conocimiento adquirido para resolver problemas y poder crear su propio conocimiento. La taxonomía de Bloom fue diseñada por Benjamin Bloom en 1956 en la Universidad de Chicago. Por otro lado, la presente investigación estuvo basada en los postulados de Barrows (1986), los cuales expresan que el Aprendizaje Basado en Problemas (ABP), es una estrategia de enseñanza, en la cual se utiliza un procedimiento inquisitivo y activo para el aprendizaje con el propósito de resolver situaciones relevantes de la vida diaria. La estrategia de enseñanza ABP en la clase de matemática implica la colaboración activa del estudiante con el maestro y sus pares.

\subsection{Aprendizaje significativo}

Ausubel (2002) definió el aprendizaje significativo como un proceso por medio del cual el estudiante relaciona información nueva que es relevante para el material que intenta aprender con la ya existente en su estructura cognitiva. Ausubel expone que para que esa relación ocurra es necesario que se den, de manera simultánea, las siguientes condiciones: el contenido que se ha de aprender debe tener sentido lógico, el contenido debe articularse con sentido psicológico en la estructura cognoscitiva del aprendiz, mediante su anclaje en los conceptos previos y el estudiante debe tener deseos de aprender, es decir, que su actitud sea positiva hacia el aprendizaje.

Siendo el aprendizaje significativo parte sustantiva de la estrategia de enseñanza ABP y con base en lo expuesto por Ausubel (2002) la investigadora elaboró un marco teórico que muestra la secuencia del aprendizaje significativo desarrollado en tres fases. El mismo se ilustra en la figura 1. La fase inicial, es la fase en la cual el estudiante percibirá hechos 
aislados conceptualmente. La segunda fase es la fase intermedia, en la cual el estudiante tendrá la oportunidad para reflexionar y poder organizar las ideas. La tercera fase, conocida como fase final, es la fase en la cual el estudiante es capaz de retener el nuevo conocimiento.

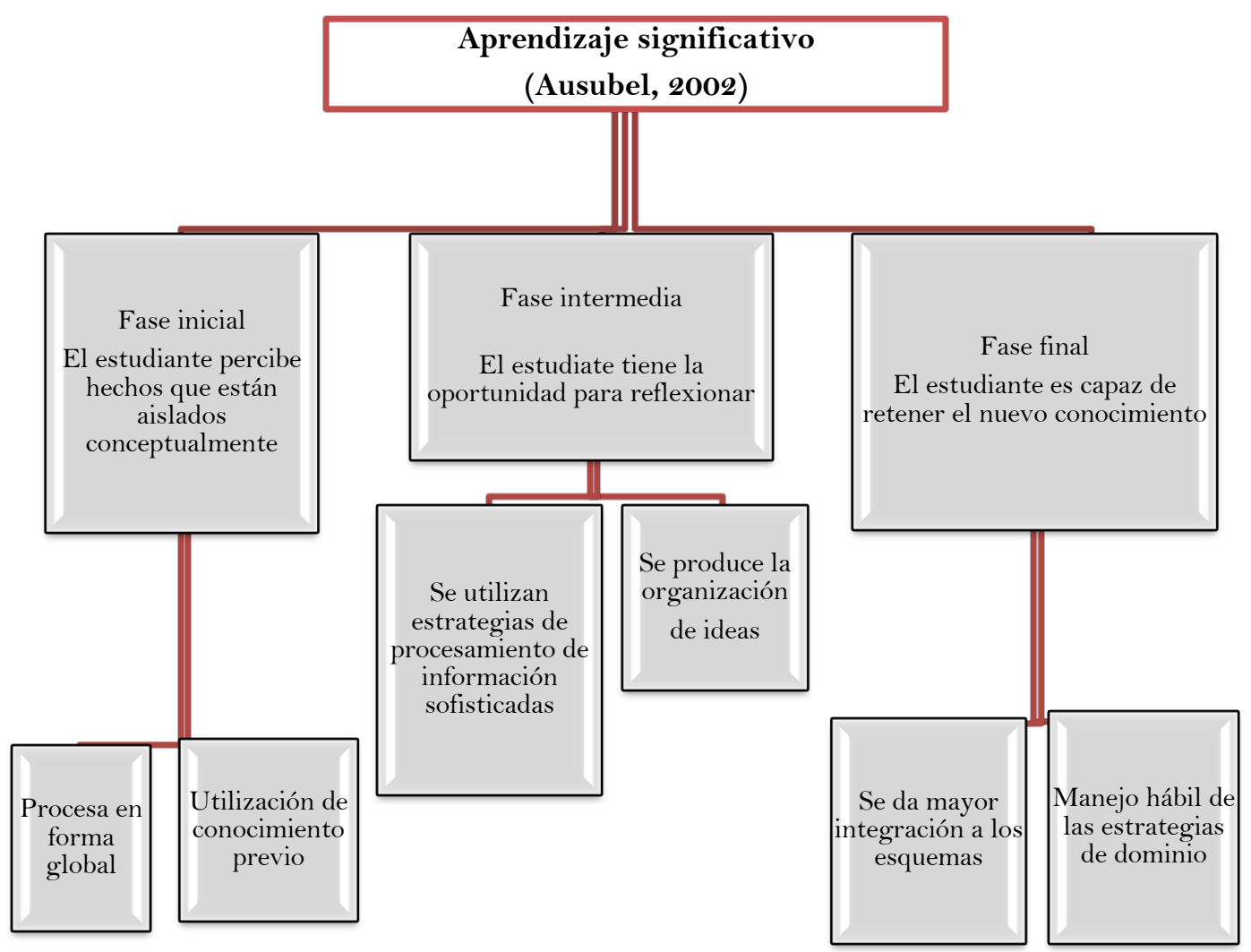

Figura 1. Marco teórico de la secuencia del aprendizaje significativo de Ausubel (2002)

Fuente: Elaboración propia.

\subsection{Taxonomía de Benjamin Bloom}

La Taxonomía de Bloom revisada en el 2001 por Anderson y Krathwohl, clasificó y ordenó el aprendizaje, de forma tal que, facilita la acción planificadora de los docentes. Uno de los aspectos clave de esta revisión es el cambio de los sustantivos de la propuesta original a verbos, para significar las acciones correspondientes a cada categoría. Otro aspecto fue considerar la síntesis con un criterio amplio el cual conlleva crear. Además, se modificó la secuencia en que se presentan las distintas categorías. El nivel cognoscitivo incluye las sub áreas recordar, comprender, aplicar, analizar, evaluar y sintetizar. Para fines de esta investigación se integró al marco conceptual los niveles de comprensión y de análisis de la Taxonomía de Bloom. La figura 2 ilustra los componentes de la Taxonomía de Bloom. 


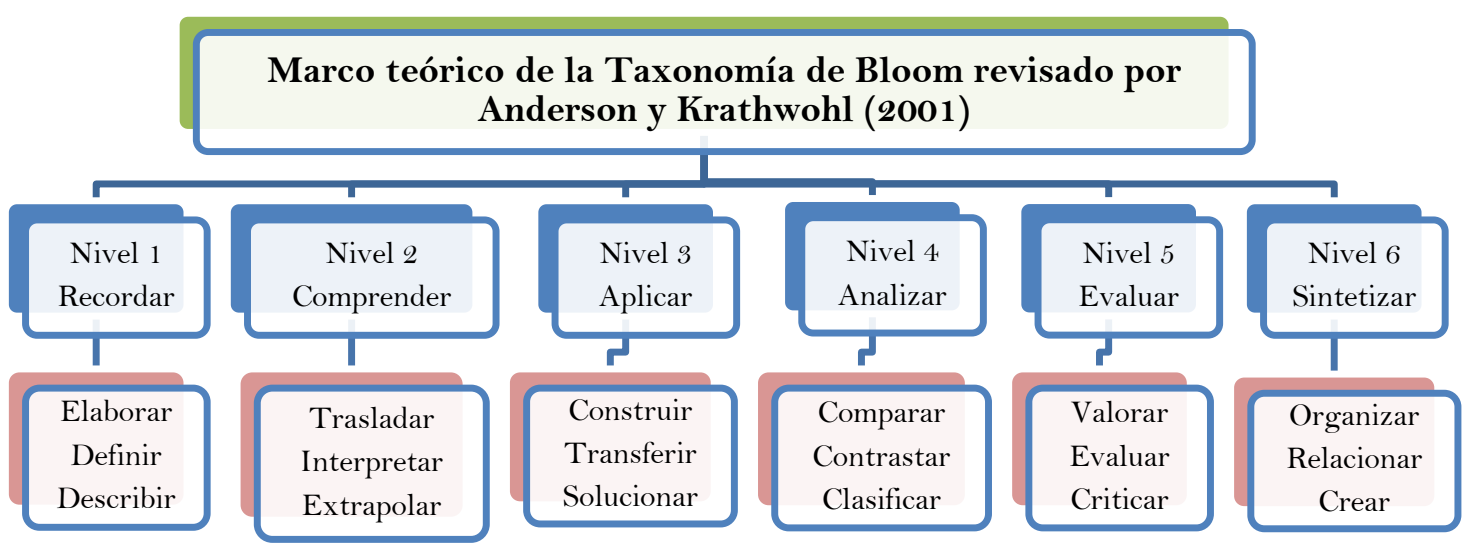

Figura 2. Marco teórico de la Taxonomía de Bloom Fuente: Elaboración propia.

\subsection{Aprendizaje Basado en Problemas}

La Universidad de McMaster, situada en Hamilton, Canadá, introdujo el Aprendizaje Basado en Problemas (ABP) en 1969, en la enseñanza de la medicina bajo el liderazgo de Howard Barrows. Más tarde, a principios de la década de 1980, Mercer University, en los Estados Unidos (EEUU) adoptó un currículo basado en la estrategia de enseñanza ABP y a finales de la misma década, lo hizo también la Escuela de Medicina de la Universidad de Harvard en EEUU. Las escuelas de medicina en la Universidad de Limburg en Maastricht (Holanda), la Universidad de Newcastle (Australia), y la Universidad de Nuevo México (EEUU), adaptaron el modelo de McMaster.

La estrategia de enseñanza ABP está sustentada con la teoría del aprendizaje significativo de Ausubel (2002). De acuerdo con esta teoría, en el diseño, la elaboración y la aplicación del ABP, es de gran importancia el contexto y presentación del problema para lograr un aprendizaje significativo.

Según Moreira (2003), el uso de la estrategia ABP en el proceso de enseñanza debe estar basado en iniciar la discusión de un problema y el aprendizaje significativo se irá adquiriendo a través de la experiencia de trabajar sobre ese problema. Esto estimulará el autoaprendizaje y la práctica del estudiante al enfrentarlo a situaciones reales y al identificar sus deficiencias de conocimiento. De la misma forma, el autor expone que el trabajo colaborativo promueve un aprendizaje más significativo del que se logra a través del trabajo individual.

El uso de la estrategia ABP para enseñar y aprender, promueve en el estudiante la investigación de situaciones planteadas y motiva la interacción entre los estudiantes y el material educativo; entre los estudiantes, entre los estudiantes y el profesor, lo que permite la negociación de significado y la transferencia de contenidos a situaciones nuevas. El ABP permite que los estudiantes adquieran un aprendizaje significativo en un contexto metodológico activo-participativo, que tiene como fin lograr que los estudiantes aprendan a través de estrategias de aprendizaje profundas, que lleven a un aprendizaje significativo y funcional donde se pueda aplicar y transferir lo aprendido a situaciones nuevas (Moreira, 2003).

Omstein y Leviene (1985) expresaron que la estrategia de enseñanza ABP era una propuesta educativa innovadora, que se caracteriza en que el aprendizaje está centrado en el estudiante. La misma promueve el desarrollo de una serie de habilidades y competencias 
indispensables en el entorno profesional. Con el ABP se pretende que el estudiante aprenda a desenvolverse como un profesional capaz de identificar y resolver problemas, de comprender el efecto de su propia actuación profesional y las responsabilidades éticas que implica, de interpretar datos y diseñar estrategias; y en relación con todo ello, ha de ser capaz de poner en práctica el conocimiento teórico que es adquirido en su formación.

De acuerdo con Barrows (1986), el ABP, es una estrategia de enseñanza, en la cual se utiliza un procedimiento inquisitivo y activo para el aprendizaje con el propósito de resolver situaciones relevantes de la vida diaria. Barrows (1986) estableció que el ABP ocurre en un entorno de aprendizaje colaborativo entre pares, en el cual el maestro sirve como facilitador del proceso de enseñanza y aprendizaje. Además, expone que esta estrategia se centra en el estudiante y sus intereses, en la cual se investiga y luego se buscan soluciones a través de la creación de proyectos que estimulen la capacidad de pensamiento crítico, la creatividad, el aprendizaje colaborativo y el análisis. Por consiguiente, el estudiante tiene la libertad de personalizar su aprendizaje y de expresar sus conocimientos de manera contextual.

Para Bernabeu y Cónsul (2004) el ABP es una estrategia de enseñanza, en la cual, tanto el adquirir el conocimiento y el desarrollo de habilidades y actitudes tienen la misma importancia. Dicha estrategia requiere de grupos pequeños de estudiantes, que analicen y resuelvan un problema con la ayuda de un maestro. Son los estudiantes quienes determinan sus necesidades de aprendizaje, logran su propio aprendizaje y trabajan de forma colaborativa. Un maestro que utiliza la estrategia de enseñanza ABP debe ser capaz de ayudar a los estudiantes a definir con claridad, sintetizar, defender opiniones, promover el pensamiento crítico de los estudiantes a través del cuestionamiento y favorecer la participación de todos los estudiantes en el grupo.

Desde que, en el año 2005, la Universidad de Murcia comenzó la convocatoria de proyectos de innovación educativa, se desarrollaron una serie de actividades encaminadas a fomentar acciones dirigidas a mejorar la calidad del aprendizaje. En este contexto, un grupo de profesores de esta universidad desarrolló diferentes estrategias de enseñanza basadas en el ABP. Boss (2011) a su vez, estableció que España no había sido la excepción en introducir el uso de la estrategia del ABP. Según Velázquez y Figarella (2012), la estrategia de enseñanza ABP tiene implícito en su dinámica de trabajo el desarrollo de habilidades, actitudes y valores que pretenden beneficiar personal y profesionalmente al estudiante. Basándose en lo anteriormente expuesto por los autores, la investigadora elaboró un esquema en el que se representan las fases que contempla el ABP. El mismo se ilustra en la figura 3. 


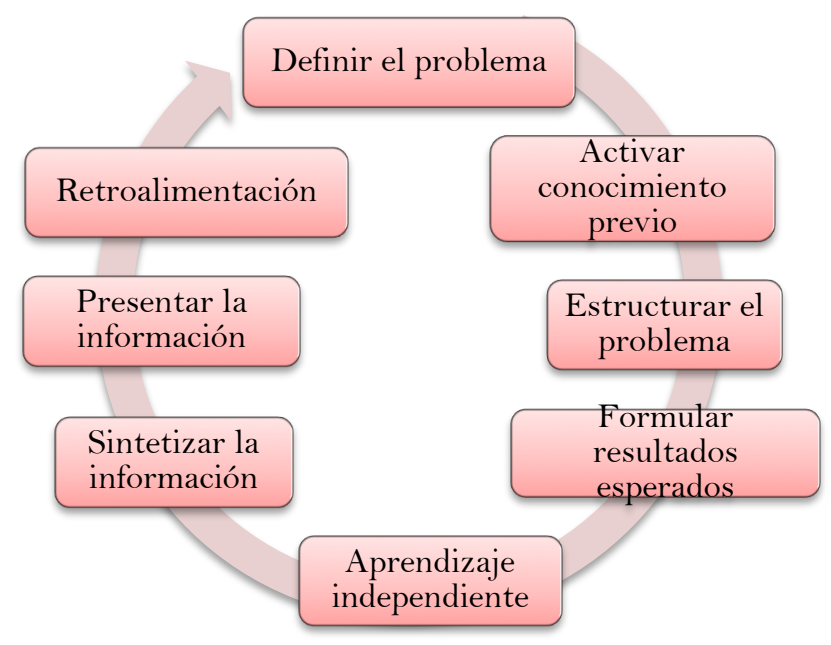

Figura 3. Marco teórico de las fases del proceso de ABP Fuente: Elaboración propia.

\subsection{Marco conceptual}

En la figura 4, se presenta el marco conceptual de la integración de la estrategia de enseñanza ABP (Barrows, 1986) en el desarrollo de las destrezas de comprensión y análisis (Bloom, 1956) para lograr un aprendizaje significativo (Ausubel, 2002), en estudiantes de décimo grado. El utilizar la estrategia de enseñanza ABP durante el proceso de enseñanza y aprendizaje de las matemáticas, permite que los estudiantes puedan desarrollar con mayor eficacia las destrezas de comprensión y análisis. Esto a su vez logrará que puedan obtener un aprendizaje significativo, siendo esta parte sustantiva del ABP y puedan mejorar su desempeño académico. Con el uso de la Taxonomía de Bloom, se pretende que los estudiantes lleguen a desarrollar habilidades que les permitan utilizar el conocimiento adquirido para resolver problemas matemáticos de la vida diaria y poder crear su propio conocimiento. Esta taxonomía es jerárquica, ya que presume que el aprendizaje a niveles superiores depende del conocimiento y habilidades de ciertos niveles inferiores. 
La estrategia de enseñanza ABP en el desarrollo de las destrezas de comprensión y análisis para lograr un aprendizaje significativo en las matemáticas

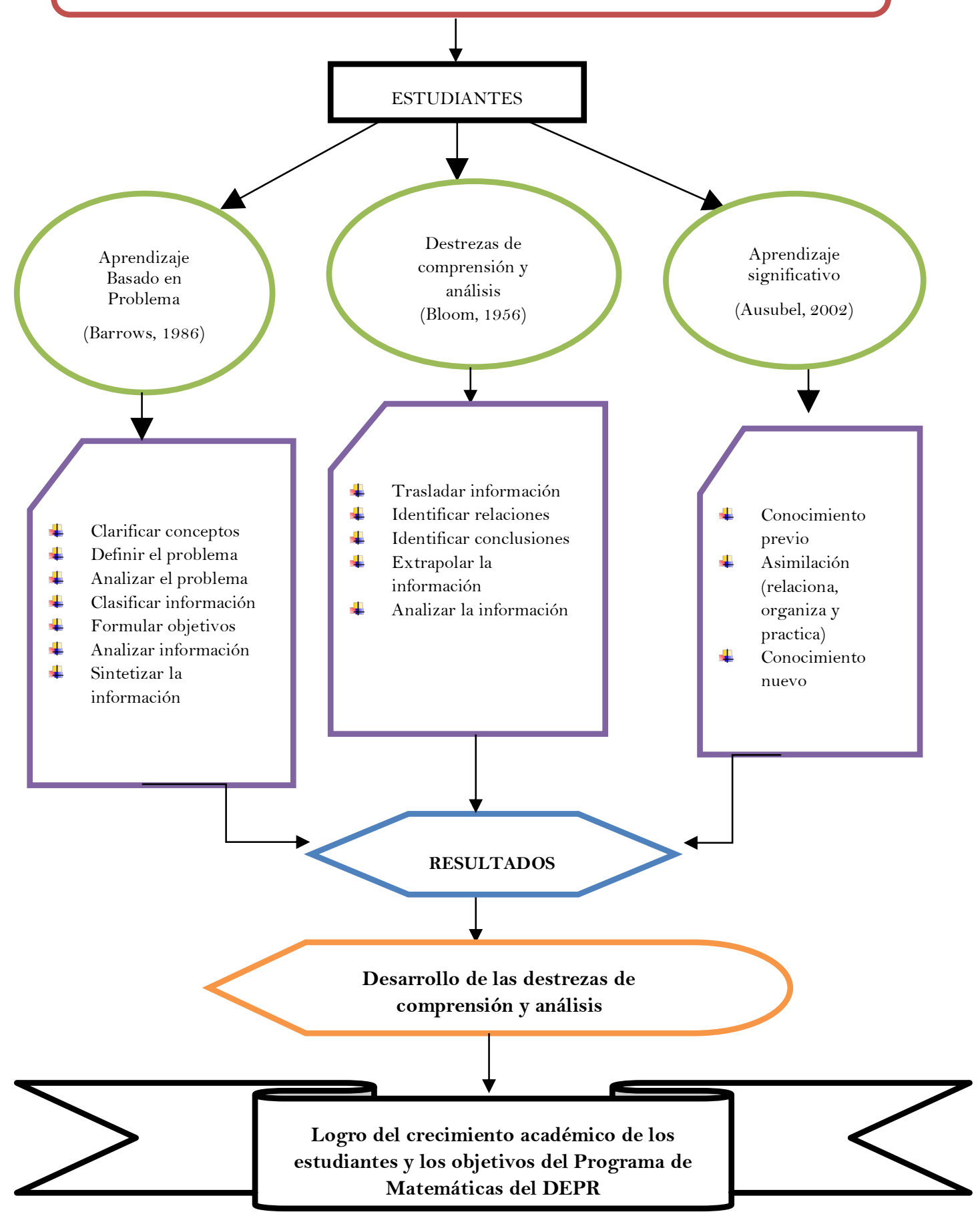

Figura 4. Marco conceptual: Integración del ABP, destrezas de comprensión y análisis para un aprendizaje significativo en las matemáticas

Fuente: Elaboración propia. 


\section{Método}

En esta investigación, se utilizó un enfoque cuantitativo. Asimismo, el diseño que se utilizó fue cuasi experimental con dos grupos independientes con preprueba y posprueba. La investigación incluyó un grupo control (GC) y un grupo experimental (GE). La estrategia de enseñanza que se utilizó con el GC fue una enseñanza centrada en el maestro, en la cual el estudiante fue receptor de contenido educativo y luego transfirió lo aprendido en un sistema de evaluación que mayormente estimula la capacidad memorística del estudiante. Por otro lado, al GE, se le administró el tratamiento (X). El mismo consistió en utilizar la estrategia de enseñanza ABP durante el estudio del tema de estadística descriptiva.

Cuadro 2. Diseño de investigación

\begin{tabular}{cccc}
\hline GruPOS & PrePruEBa & Tratamiento & Posprueba \\
\hline Control $(\mathrm{GC})$ & $\mathrm{O}_{1}$ & $\overline{\mathrm{BB}}$ & $\mathrm{O}_{2}$ \\
Experimental $(\mathrm{GE})$ & $\mathrm{O}_{1}$ & $\mathrm{O}_{2}$ \\
\hline
\end{tabular}

Fuente: Elaboración propia.

\subsection{Población y muestra}

La población de esta investigación fueron todos los estudiantes de décimo grado de una escuela del centro de la Isla. El diseño de la investigación fue cuasi experimental, ya que los grupos estaban conformados antes de la investigación. La muestra seleccionada para llevar a cabo esta investigación estuvo compuesta por dos grupos de estudiantes de décimo grado, los cuales fueron seleccionados por disponibilidad y conveniencia. El GC estuvo compuesto por 25 estudiantes y el GE por 23 estudiantes. Los grupos fueron heterogéneos, con estudiantes de corriente regular y estudiantes del programa de educación especial en los grupos seleccionados. Se excluyó el grupo avanzado por no haber grupo de comparación.

Cuadro3. Muestra de la investigación

\begin{tabular}{lcc}
\hline \multicolumn{1}{c}{ GrUPO } & TRATAMIENTO & CANTIDAD DE ESTUDIANTES \\
\hline Control & Ninguno & 25 \\
Experimental & ABP & 23 \\
\hline Total & - & 48 \\
\hline
\end{tabular}

Fuente: Elaboración propia.

\subsection{Instrumentos de investigación}

Para recopilar los datos necesarios de esta investigación se utilizó una prueba diseñada por la investigadora. La prueba estuvo alineada a los estándares de contenido y expectativas de décimo grado del Programa de Matemáticas del DEPR.

\subsubsection{Prueba}

La prueba fue diseñada para el tema de estadística descriptiva, específicamente los siguientes temas: moda, media, mediana, frecuencia, rango, sesgo, varianza, desviación estándar, distribución normal, probabilidad y por ciento. La misma constó de 30 reactivos de selección múltiple. Cada reactivo tuvo cinco alternativas en la cual solo una de estas era la correcta. El instrumento utilizado en esta investigación fue validado por un panel de cinco expertos para certificar la validez de contenido y de constructo. Los integrantes del panel poseían vasto dominio de la materia de matemáticas. Además, se consideró la preparación académica de cada uno de ellos. Una vez validada la prueba y luego de obtener 
los permisos correspondientes se llevó a cabo un estudio piloto para determinar el coeficiente de confiabilidad de la referida prueba.

\subsection{Procedimiento}

La investigación fue autorizada por el Departamento de Educación de Puerto Rico (DEPR) y por la Junta de Revisión Institucional (JRI) de la Pontificia Universidad Católica de Puerto Rico. Posterior a esto se llevó a cabo la explicación de la investigación, en las escuelas seleccionadas, a los directores, maestros cooperadores, estudiantes participantes y a los padres de estos. Se obtuvo el consentimiento y asentimiento informado de todos los participantes.

\subsubsection{Estudio piloto}

Una vez obtenida la autorización de la JRI para llevar a cabo la investigación se procedió a realizar el estudio piloto para determinar el coeficiente de confiabilidad del instrumento. El coeficiente de confiabilidad de acuerdo con Hernández-Sampieri, Fernández-Collado y Batista-Lucio (2010), se refiere a la consistencia de los resultados de un instrumento de medición. Dicho coeficiente fue de 0,904.

\subsubsection{Administración de la preprueba}

Una vez obtenidos los permisos correspondientes para llevar a cabo la investigación, se administró la preprueba tanto al grupo control (GC) como al grupo experimental (GE). Para mantener la pureza de la investigación, la preprueba fue ofrecida y evaluada por un maestro cooperador en la investigación.

\subsubsection{Administración de tratamiento}

Se aplicó el tratamiento: integración de la estrategia de enseñanza Aprendizaje Basado en Problema en el desarrollo de las destrezas de comprensión y análisis de la estadística descriptiva. El mismo se llevó a cabo durante cinco periodos de cincuenta minutos, por cinco semanas mientras se desarrollaron principalmente, las destrezas de comprensión y análisis.

La maestra pidió a los estudiantes que trajeran posibles temas que tuvieran relación con sus experiencias previas o su futuro profesional. Los mismos debían ser atractivos, interesantes y motivadores. Algunos de los temas que los estudiantes expusieron fueron: tasa de desempleo en Puerto Rico, uso de la tecnología en los jóvenes de la escuela superior en Puerto Rico, emigración en Puerto Rico, efectos del huracán María en Puerto Rico y hábitos de estudio de los estudiantes de educación secundaria en Puerto Rico, entre otros. Se formaron cinco grupos de cuatro estudiantes y un grupo de tres estudiantes y se identificaron los roles de los estudiantes con cada una de sus funciones: moderador, colaborador, creativo, relacionista y evaluador.

Cada grupo de estudiantes seleccionó el problema que iba a trabajar. Con la maestra como guía, los estudiantes indagaron en el problema y crearon un cuestionario con preguntas relacionadas al tema seleccionado, con el cual iban a recopilar los datos necesarios para ser analizados. Determinaron qué información conocían y sintetizaron lo que sabían sobre el mismo. Luego, la maestra fue trabajando destrezas dentro del tema de la estadística descriptiva según estipuladas en el mapa curricular del Programa de Matemáticas del DEPR tales como: media, moda, mediana, rango, frecuencia, varianza, desviación estándar, distribución normal, por ciento y probabilidad. 
Los estudiantes fueron investigando con los conocimientos previos que disponían y fueron identificando los conocimientos que debían adquirir. Luego, establecieron sus necesidades de aprendizaje, fijaron sus objetivos y los recursos de información a utilizar y se distribuyeron las tareas. Cada estudiante, luego presentó su parte a su equipo de trabajo. Al finalizar la enseñanza de las diferentes destrezas y las diferentes tareas, los estudiantes presentaron de forma oral y escrita, los trabajos diseñados y elaborados a través del uso de la estrategia de enseñanza ABP.

\subsubsection{Administración de posprueba}

Al finalizar la administración del tratamiento, tanto los estudiantes del GC como el GE, contestaron la posprueba, siendo ésta el mismo instrumento utilizado para la preprueba. Para mantener la pureza de la investigación, la posprueba fue ofrecida y evaluada por un maestro cooperador en la investigación.

\section{Resultados}

Para determinar el comportamiento de las variables y el análisis estadístico a utilizarse en la investigación, se llevó a cabo la Prueba de Normalidad Shapiro-Wilk, con un nivel de significancia de $\mathrm{p}>0,05$. Como hipótesis nula se estableció que la muestra tiene una distribución normal. Los valores, tanto el GC $(0,495)$ como del GE $(0,302)$ fueron mayores a p >0,05, lo que implica que no se puede rechazar la hipótesis nula. Esto quiere decir que, la muestra tiene una distribución normal. De la misma forma, la igualdad de varianza, también con un nivel de significancia de $\mathrm{p}>0,05$ se cumplió tanto para la preprueba $(0,363)$ como para la posprueba $(0,399)$.

Por lo anteriormente expuesto, la información recopilada mediante la preprueba y la posprueba se analizó mediante la prueba $t$ para grupos independientes, para determinar probable diferencia entre las medias de la preprueba y las de la posprueba del GC y el GE. Asimismo, se utilizó la prueba $t$ para muestras pareadas, para determinar probables diferencias dentro de los grupos respecto a las puntuaciones obtenidas en la preprueba y en la posprueba. Las hipótesis se comprobaron a un nivel de significancia o alfa $(\alpha)$ de 0,01 y 0,05. Todos los análisis se realizaron a través del uso del programado estadístico Statistical Package for the Social Science (SPSS), Versión 24.

\subsection{Resultados de la Prueba t para grupos independientes (Preprueba)}

En el cuadro 4 se muestran los resultados del análisis estadístico realizado de la Prueba $t$ de la preprueba de los GC y GE.

Cuadro 4. Resultados de la prueba t de los GC y GE

\begin{tabular}{rrrrccccc}
\hline \multicolumn{2}{c}{ GRUPO } & $\boldsymbol{N}$ & $\boldsymbol{\sigma}$ & DE & EM & $\boldsymbol{T}$ & $\boldsymbol{D} \boldsymbol{F}$ & $\boldsymbol{P}$ \\
\hline GC & Preprueba & 25 & 7,52 & 2.417 &, 483 & & & \\
& & & & & & $-1,570$ & 46 &, 123 \\
GE & Preprueba & 23 & 8,70 & 2.771 &, 578 & & & \\
\hline
\end{tabular}

Nota. $\mathrm{n}=$ número de la muestra, $\sigma=$ media, $\mathrm{DE}=$ desviación estándar, $\mathrm{EM}=$ error en la media, $\mathrm{t}$ $=$ valor de la prueba $\mathrm{t}, \mathrm{df}=$ grados de libertad, $\mathrm{p}=$ nivel de significancia $(\mathrm{p}<0,05)$

Fuente: Elaboración propia.

De acuerdo con la información del cuadro 4 , el valor $p=0,123$, lo que significa que no existe diferencia significativa $(\mathrm{p}>0,05)$ entre las puntuaciones de la preprueba del GE y el GC. Como consecuencia, no existe diferencia en las puntuaciones de la preprueba entre 
los estudiantes a los que se le aplicó el tratamiento (GE) y a los que no se les aplicó el tratamiento $(\mathrm{GC})$.

\subsection{Resultados de la Prueba t pareada para el GE}

En el cuadro 5 se muestran los resultados del análisis estadístico realizado de la Prueba $t$ para muestras pareadas del GE.

Cuadro 5. Resultados de la Prueba t del grupo experimental (GE)

\begin{tabular}{cccccccc}
\hline $\begin{array}{c}\text { GRUPO } \\
\begin{array}{c}\text { EXPIMENTAL } \\
(\text { GE) }\end{array}\end{array}$ & $\mathbf{N}$ & $\boldsymbol{\sigma}$ & DE & $\mathbf{E M}$ & $\mathbf{T}$ & $\mathbf{D F}$ & $\mathbf{P}$ \\
\hline Preprueba & 23 & 8,70 & 2,771 &, 578 & & & \\
Posprueba & 23 & 21,13 & 4,751 &, 991 & & &, 000 \\
\hline
\end{tabular}

$\mathrm{n}=$ número de la muestra, $\sigma=$ media, $\mathrm{DE}=$ desviación estándar, $\mathrm{EM}=$ error en la media, $\mathrm{t}=$ valor de la prueba $\mathrm{t}, \mathrm{df}=$ grados de libertad, $\mathrm{p}=$ nivel de significancia $(\mathrm{p}<.05)$

Fuente: Elaboración propia.

De acuerdo con la información del cuadro 5 , el valor de $p=0,000$. Por lo tanto, existe diferencia significativa $(p<0,05)$ en el GE entre las puntuaciones obtenidas en la preprueba y la posprueba por parte del GE. La puntuación de la media de la preprueba fue de 8,70 puntos y de 21,13 puntos en la posprueba. Por lo tanto, se acepta la hipótesis de investigación: existe diferencia significativa en las puntuaciones de la preprueba y posprueba obtenida por los estudiantes del GE.

\subsection{Resultados de la Prueba t pareada para el grupo control}

En el cuadro 6 se muestran los resultados del análisis estadístico realizado de la Prueba $t$ para muestras pareadas del GC.

Cuadro 6. Resultados de la Prueba t pareada del grupo control

\begin{tabular}{cccccccc}
\hline $\begin{array}{c}\text { GruPO } \\
\text { CoNTROL } \\
(\mathbf{G C})\end{array}$ & N & $\boldsymbol{\sigma}$ & DE & EM & T & DF & P \\
\hline Preprueba & 25 & 7,52 & 2.147 &, 483 & & & \\
Posprueba & 25 & 13,44 & 3,959 &, 792 & & & \\
\hline
\end{tabular}

Fuente: Elaboración propia.

De acuerdo con la información de el cuadro 6 , el valor $p=0,000$. Por lo tanto, existe diferencia significativa $(p<0,05)$ entre las puntuaciones obtenidas en la preprueba y la posprueba por el grupo control. La puntuación media de la preprueba fue de 7,52 puntos y de 13,44 puntos en la posprueba. Esta diferencia reflejó un aumento significativo en los resultados de la posprueba. Por consiguiente, se acepta la quinta hipótesis de investigación. Como consecuencia, existe evidencia para rechazar la hipótesis nula.

\subsection{Resultados de la Prueba t para grupos independientes (Posprueba)}

En el cuadro 7 se presentan los resultados del análisis estadístico efectuado respecto a las puntuaciones obtenidas en la posprueba por el GC y del GE.

De acuerdo a el cuadro 7 , el valor de $\mathrm{p}=0,000$, por lo tanto, existe diferencia significativa $(\mathrm{p}<0,05)$ entre las puntuaciones de la posprueba del GE y del GC. La media de las puntuaciones obtenida en la posprueba del grupo control fue de 13,44 puntos y de 21,13 
puntos para el GE. Los estudiantes a los cuales se les aplicó el tratamiento ABP obtuvieron puntuaciones significativamente más altas que los estudiantes a los cuales no se le aplicó el tratamiento. Por lo tanto, se acepta la hipótesis de investigación.

Cuadro 7. Resultados de la Prueba t para grupos independientes: puntuaciones obtenidas en la posprueba del GC y del GE

\begin{tabular}{llccccccc}
\hline & GRUPO & N & $\boldsymbol{\sigma}$ & DE & EM & T & DF & P \\
\hline GC & Posprueba & 25 & 13,44 & 3,959 &, 792 & & & \\
GE & Posprueba & 23 & 21,13 & 4,751 &, 991 & & & ,000 \\
\hline
\end{tabular}

Fuente: Elaboración propia.

\section{Discusión y conclusiones}

El propósito de esta investigación fue determinar el efecto de la estrategia de enseñanza $\mathrm{ABP}$ en el desarrollo de las destrezas de comprensión y análisis en la estadística descriptiva en estudiantes de décimo grado en una escuela en un distrito escolar del centro de Puerto Rico. Para llevar a cabo la discusión de los hallazgos, se tomaron en consideración la implementación de la estrategia de enseñanza ABP, la teoría del aprendizaje significativo de Ausubel (2002), la Taxonomía de Bloom (1956) y los postulados del ABP de Barrows (1986).

El hallazgo de la diferencia en las puntuaciones en la posprueba de los estudiantes del GE y los estudiantes del GC coincidieron con los resultados de Padilla (2009) cuya investigación pretendió determinar el efecto de la estrategia de enseñanza del ABP en el desarrollo de las destrezas de pensamiento y el aprovechamiento académico de los estudiantes en el nivel universitario y reveló que el uso de la estrategia del ABP tiene un efecto positivo en el aprovechamiento académico y en el desarrollo de las destrezas de comprensión y análisis de los estudiantes. De la misma forma, este hallazgo coincidió con los resultados de la investigación de Pantoja y Covarrubias (2013), quienes pretendían determinar cómo la estrategia de ABP muestra la promoción de habilidades de pensamiento necesarias para el aprendizaje significativo en los diferentes contenidos y los cuales demostraron que la integración de la estrategia de enseñanza ABP aumentó significativamente el desempeño académico de los estudiantes, la participación en equipos de trabajo y la motivación por la estrategia empleada. Además, los autores exponen que el $\mathrm{ABP}$ es una estrategia didáctica complementaria que potencia estrategias más tradicionales. A su vez, los resultados de la diferencia en las puntuaciones en la posprueba de los estudiantes del GE y los estudiantes del GC coincidieron con Fuentes y Pérez (2013), cuya investigación tuvo como objetivo el determinar el efecto del ABP en el aprendizaje logrado por estudiantes y cuyos resultados de su investigación, demostraron diferencia significativa en el rendimiento académico, nivel de comprensión y análisis, en los estilos de aprendizajes y antecedentes académicos previos en los estudiantes del GE.

Los resultados sobre la diferencia en las puntuaciones en la posprueba de los estudiantes del GE y los estudiantes del GC contrastaron con el de Savery y Duffy (1996) quienes indicaron que la comprensión se da como resultado de la interacción con el ambiente, y no necesariamente por el uso de una estrategia de enseñanza en específico. De la misma forma, contracta con el de Labra (2011), cuya investigación tuvo como propósito determinar el efecto de competencias desarrolladas basada en los objetivos de los Módulos $\mathrm{ABP}$ en su quehacer pedagógico y quien expuso que no se puede atribuir directamente el 
logro de ciertos objetivos exclusivamente al ABP, ya que las características personales son una variable que incide sobre el desempeño.

Por otro lado, el hallazgo de la diferencia en las puntuaciones en la preprueba y la posprueba de los estudiantes del GE sobre el desarrollo de las destrezas de comprensión y análisis de la estadística descriptiva coincidió con el de Carmona, Conesa y Ros (2014), cuyo objetivo en su investigación fue determinar el efecto de la aplicación de la estrategia de enseñanza $\mathrm{ABP}$ en el proceso de enseñanza y aprendizaje de los estudiantes. Los autores expusieron que integrar el ABP en el proceso de enseñanza y aprendizaje resulta en una experiencia creativa y valorada por los estudiantes. A su vez, los hallazgos coincidieron con Soto, Figarella y González (2014) que pretendían determinar cómo la estrategia de ABP promovía el desarrollo de la alfabetización crítica y de competencias en estudiantes de tercer grado, así como las destrezas de análisis, inferir, deliberar y cuestionar, entre otros y quienes concluyeron que el ABP es una excelente estrategia para el desarrollo de las destrezas de comprensión y que la misma promueve el desarrollo integral de los estudiantes. Estos resultados coinciden, además, con los de Heredia y Olivares (2012), quienes realizaron una investigación que tuvo como objetivo comparar el desarrollo de niveles de pensamiento crítico de estudiantes que utilizaron la estrategia de enseñanza $\mathrm{ABP}$, con estudiantes que no fueron expuestos a esta estrategia y quienes encontraron que entre las destrezas que el ABP contribuye a desarrollar se incluyen la comprensión, el análisis, la inferencia y la evaluación.

No obstante, los hallazgo de la diferencia en las puntuaciones en la preprueba y la posprueba de los estudiantes del GE contrastan con los resultados de la investigación de Gutiérrez (2012) cuyo objetivo fue realizar un análisis comparativo respecto al efecto de dos estrategias de enseñanza y el aprendizaje colaborativo y los objetos de aprendizaje reutilizables en un Entorno Virtual de Aprendizaje, en el logro del aprendizaje de los estudiantes y quien encontró que no existen diferencias significativas en las medias del rendimiento académico de los estudiantes que fueron expuestos al ABP y los que fueron expuestos a enseñanza tradicional. Esto es, según el autor, que no hubo diferencia significativa en los niveles de comprensión y asimilación de ambos grupos.

La estrategia de enseñanza ABP utilizada como tratamiento experimental en la presente investigación se fundamentó en la teoría del aprendizaje significativo de Ausubel (2002), la Taxonomía de Bloom (1956) y los postulados del ABP de Barrows (1986). Los hallazgos de la misma avalan los supuestos y los postulados de Barrow (1986) respecto al ABP. Según Barrows, el ABP es una estrategia centrada en el estudiante y debe implantarse en un ambiente de aprendizaje colaborativo, en el cual el maestro sirve como facilitador del proceso de enseñanza y aprendizaje. Esta estrategia a su vez, permite al estudiante investigar y buscar posibles soluciones mediante la creación y desarrollo de estrategias que estimulen el pensamiento crítico, la comprensión, la creatividad, el aprendizaje colaborativo y el análisis. El uso del ABP en esta investigación condujo a la colaboración entre pares y al énfasis en la comunicación oral y escrita. Tal uso contribuyó, además, a relacionar el nuevo contenido del tema de estadística descriptiva con los conceptos matemáticos ya existentes en la estructura cognitiva de los estudiantes lo que facilitó el aprendizaje del nuevo contenido. En este caso, de acuerdo con los resultados de la investigación, se infiere que el ABP aportó a la motivación para lograr el desarrollo de las destrezas de comprensión y análisis de la estadística en los estudiantes de décimo grado del GE. 
La integración de la Taxonomía de Bloom (1956) al proceso de enseñanza y aprendizaje permitió que los estudiantes desarrollaran y adquirieran habilidades que les permitieran utilizar el conocimiento adquirido para resolver problemas y poder crear su propio conocimiento. En este caso, de acuerdo con los resultados de la investigación llevada a cabo, se infiere que las actividades integradas en el ABP promovieron el desarrollo de las destrezas de comprensión y análisis de la estadística descriptiva en los estudiantes, lo que, a su vez, contribuyó a aumentar su nivel de conocimiento respecto a la estadística descriptiva.

Para aportar a la base teórica que sustenta los resultados de la diferencia significativa entre los estudiantes del GC y GE, Bloom (1986) establece que aquellas personas que trabajan con pares son capaces de entender cómo lograron realizar una tarea. Por lo tanto, de acuerdo con los resultados de la investigación, el énfasis del ABP fundamentado en el trabajo colaborativo, influyó en el nivel de conocimiento que obtuvieron los estudiantes del GE.

El aumento significativo en las puntuaciones de la posprueba del GE al compararlas con las del GC permitió concluir que el uso de la estrategia de enseñanza ABP tiene efecto en el nivel de desarrollo de las destrezas de comprensión y análisis relacionadas a la estadística descriptiva. Además, los análisis estadísticos realizados con los datos de la preprueba y posprueba del GE evidencian que la enseñanza de las matemáticas fundamentada en el ABP en el desarrollo de las destrezas de comprensión y análisis en la estadística descriptiva aumenta significativamente el nivel de conocimiento que tienen los estudiantes de décimo grado de la estadística descriptiva.

De la misma forma, los estudiantes a los cuales se le aplicó el tratamiento (GE) ABP tuvieron la oportunidad de trabajar en equipo, ejercer diferentes roles y de seleccionar el tema sobre el cual trabajarían, por lo que se concluye que el tratamiento contribuyó al aumento significativo en el rendimiento de los estudiantes del grupo experimental en la posprueba (valor añadido).

\section{Referencias}

American Psychological Association. (2016). An introduction to APA Style: A Student guide for the $6^{\text {sh }}$ edition of the APA Publication Manual. Washington, DC: APA.

Ausubel, D. (2002). Adquisición y retención del conocimiento: Una perspectiva cognitiva. Barcelona: Ediciones Paidós Ibérica.

Barrows, H. (1986). Taxonomy of problem based learning methods. Medical Education, 20, 481486.

Bernabeu, M. D. y Cónsul, M. (2004). Similitudes entre el proceso de convergencia en el ámbito de la educación de la educación superior europea y la adopción del aprendizaje por problemas en la EUI Vall d'Hebron de Barcelona. Barcelona: Barcelona ediciones.

Bloom, B. (1956). Taxonomy of educational objectives: Handbook I, The cognitive domain. Nueva York, NY: David McKay.

Boss, S. (2011). Project based learning: A short history. Recuperado de www.edutopia.org/projecbased-learning-history

Carmona, M., Conesa, M. y Ros, M. (2014). Valoración del Aprendizaje Basado en Problemas por los alumnos: Diferencias por sexo. Historias y Comunicación Social, 19(8), 725 - 734. 
Cronbach, L. J. (1951). Coefficient alpha and the internal structure of test. Psychometrika, 16(3), 297-334.

Delgado, I. (2016). El rol de la colaboración y el Aprendizaje Basado en Problemas mediante la Teoría de Actividad (CHAT): Un estudio de caso con estudiantes de noveno grado. ProQuest Dissertations and Theses, 76(9), 233-255.

Departamento de Educación de Puerto Rico. (2016). Revisión Marco Curricular del Programa de Matemática. Recuperado de http://de.gobierno.pr/files/MARCO_CURRICLAR_MATEMATICAS.pdf

Departamento de Educación de Puerto Rico. (2018). El perfil escolar. Departamento de Educación de Puerto Rico. Recuperado de http://www.de.gobierno.pr/el-perfilescolar

Fuentes, V. y Pérez, C. (2013). Estudio comparativo entre metodologías: Aprendizaje Basado en Problemas y tradicional en módulos de enseñanza. Revista Educativa Ciencias de la Salud, $10(2), 107-113$.

Gutiérrez, C. (2012). Análisis comparativo de metodologías de aprendizaje colaborativo, JIGSAW y Aprendizaje Basado en Problemas (ABP): Haciendo uso de objetos de aprendizaje reutilizables, para el aprendizaje de la geometría, en los alumnos de primero medio. Santiago de Chile: Universidad de Chile.

Heredia, Y. y Olivares, S. (2012). Desarrollo del pensamiento crítico en ambientes de Aprendizaje Basado en Problemas (ABP) en estudiantes de educación superior. Revista Mexicana de Investigación Educativa, 17(54), 759-778.

Hernández-Sampieri, R., Fernández-Collado, C. y Baptista-Lucio, P. (2010). Metodología de la investigación. Ciudad de México: Mc Graw-Hill.

Labra, P. (2011). El enfoque ABP en la formación inicial docente de la Universidad de Atacama: El impacto en el quehacer docente. Estudios Pedagógicos, 37(1), 167-185.

Moreira, M. (1993). La teoría del aprendizaje significativo de David Ausubel. Enfoques Didácticos, 1. Recuperado de https://bit.ly/33OOPCy

Ornstein, A. y Levine, D. (1985). An introduction to the foundations of education. Boston, MA: Houghton Mifflin.

Padilla, I. (2009). Efecto de la estrategia del Aprendizaje Basado en Problemas en el desarrollo de pensamiento y aprovechamiento académico de los estudiantes. Tesis doctoral, Universidad Interamericana de Puerto Rico, Puerto Rico.

Pantoja, J. y Covarrubias, P. (2013). La enseñanza en el bachillerato a partir del aprendizaje basado en problemas. Perfiles Educativos, 35(139), 93-109.

Savery, J. y Duffy, T. (1996). Problem-Based Learning: An instructional model and its constructivist framework. Educational Technology, 2(3), 135-148.

Soto, A., González, A. y Figarella, F. (2016). Aprendizaje Basado en Problemas para desarrollar destrezas de conocimiento, alfabetización y competencias ciudadanas en el nivel elemental. Revista Actualidades Investigativas en Educación, 16(3), 1-34. https://doi.org/10.15517/aie.v16i3.26063

Velázquez, L. y Figarella, F., (2012). La problematización en el aprendizaje. Tres estrategias para la creación de un currículo auténtico. Puerto Rico: Isla Negra. 


\section{Anexo I. \\ Puntuaciones de la preprueba y la posprueba del grupo control (GC) y del grupo experimental (GE}

Cuadro 8. Puntuaciones de la preprueba y posprueba del GC y GE

\begin{tabular}{|c|c|c|c|c|c|c|c|}
\hline $\begin{array}{l}\text { PARTICIPAN } \\
\text { TE }\end{array}$ & $\begin{array}{c}\text { GRUPO } \\
\text { CONTR } \\
\text { OL } \\
(\text { GC) }\end{array}$ & $\begin{array}{l}\text { PUNTUACI } \\
\text { ÓN } \\
\text { PREPRUEB } \\
\text { A }\end{array}$ & $\begin{array}{l}\text { PUNTUACI } \\
\text { ÓN } \\
\text { POSPRUEB } \\
\text { A }\end{array}$ & $\begin{array}{c}\text { PARTICIPAN } \\
\text { TE }\end{array}$ & $\begin{array}{c}\text { GRUPO } \\
\text { EXPERIMEN } \\
\text { TAL (GE) }\end{array}$ & $\begin{array}{c}\text { PUNTUACI } \\
\text { ÓN } \\
\text { PREPRUEB } \\
\text { A }\end{array}$ & $\begin{array}{c}\text { PUNTUACI } \\
\text { ÓN } \\
\text { POSPRUEB } \\
\text { A }\end{array}$ \\
\hline 1 & GC & 8 & 14 & 1 & GE & 8 & 23 \\
\hline 2 & GC & 13 & 22 & 2 & GE & 9 & 21 \\
\hline 3 & GC & 7 & 14 & 3 & GE & 11 & 23 \\
\hline 4 & GC & 6 & 10 & 4 & GE & 9 & 14 \\
\hline 5 & GC & 7 & 18 & 5 & GE & 8 & 26 \\
\hline 6 & GC & 12 & 21 & 6 & GE & 13 & 22 \\
\hline 7 & GC & 7 & 13 & 7 & GE & 11 & 25 \\
\hline 8 & $\mathrm{GC}$ & 7 & 12 & 8 & GE & 12 & 24 \\
\hline 9 & GC & 6 & 13 & 9 & GE & 11 & 23 \\
\hline 10 & GC & 9 & 15 & 10 & GE & 9 & 27 \\
\hline 11 & GC & 5 & 13 & 11 & GE & 8 & 13 \\
\hline 12 & GC & 11 & 23 & 12 & GE & 13 & 26 \\
\hline 13 & $\mathrm{GC}$ & 6 & 10 & 13 & GE & 7 & 21 \\
\hline 14 & GC & 6 & 9 & 14 & GE & 8 & 22 \\
\hline 15 & GC & 3 & 11 & 15 & GE & 12 & 25 \\
\hline 16 & GC & 6 & 16 & 16 & GE & 6 & 26 \\
\hline 17 & GC & 4 & 8 & 17 & GE & 12 & 22 \\
\hline 18 & GC & 10 & 11 & 18 & GE & 5 & 21 \\
\hline 19 & GC & 9 & 13 & 19 & GE & 7 & 14 \\
\hline 20 & GC & 5 & 12 & 20 & GE & 6 & 21 \\
\hline 21 & GC & 10 & 14 & 21 & GE & 6 & 23 \\
\hline 22 & GC & 7 & 10 & 22 & GE & 3 & 13 \\
\hline 23 & GC & 7 & 12 & 23 & GE & 6 & 11 \\
\hline 24 & GC & 8 & 9 & & & & \\
\hline 25 & GC & 9 & 13 & & & & \\
\hline
\end{tabular}

*Grupos: GC - grupo control; GE - grupo experimental

Fuente: Elaboración propia.

\section{Breve Cv de los autores}

\section{Luz C. Colón Ortiz}

Profesora de Matemáticas del Departamento de Educación de Puerto Rico. Doctora en Educación, especialidad en Currículo y Enseñanza de la Pontificia Universidad Católica de Puerto Rico, Recinto de Ponce. Maestría en Currículo y Enseñanza con especialidad en Matemáticas: Universidad Interamericana de Puerto Rico, Recinto de Barranquitas y Bachillerato en Educación Secundaria en Matemáticas de la Pontificia Universidad Católica de Puerto Rico, Recinto de Ponce. ORCID ID: https://orcid.org/0000-00025254-809X. Email: lcolonortiz1@pucpr.edu

\section{Jaime Ortiz-Vega}

Catedrático y director de la Escuela Graduada en Educación de la Pontificia Universidad Católica de Puerto Rico. Doctor en Educación: Gerencia y Liderazgo Educativo de New York University. Consultor para agencias gubernamentales y privadas en áreas de planificación estratégica, gerencia educativa, currículo y enseñanza. Miembro de la 
American Educational Research Association y de Association for Supervision and Curriculum Development, International Level. Ex presidente del Capítulo de Puerto Rico de Asociación for Supervision and Curriculum Development. Poseedor de varias publicaciones en revistas académicas. ORCID ID: https://orcid.org/0000-0003-03109362. Email: jortiz@pucpr.edu 